\title{
Relación entre el ciclo de vida de las PYMES en redes sociales y el emprendimiento en la ciudad de Guayaquil
}

\section{Relationship between the life cycle of PYMES in social networks and entrepreneurship in the city of Guayaquil}

Lucía Magdalena Pico Versoza

Universidad Internacional del Ecuador, Ecuador

Ricardo Rafael Coello Yagual

Universidad Tecnológica Empresarial de Guayaquil, Ecuador

Autor para correspondencia: lupicove@internacional.edu.ec, ricardo_mvc@ hotmail.com

Fecha de recepción: 27 de Febrero de 2017 - Fecha de aceptación: 28 de Abril de 2017

Resumen: El presente estudio busca establecer las características de la empresa emergente digital, las ventajas que tiene como organización y la relación de los Sistemas de Información Gerencial con el ciclo de vida de las Pymes. Con las nuevas tendencias y avances en la utilización de herramientas como las tecnologías de la información, se pueden predecir escenarios que permitan establecer estrategias con mayor grado de exactitud, es decir plataformas de transformación de las pequeñas y medianas empresas en organizaciones competitivas y rentables, estas herramientas de negocios como el Business Intelligence, CRM o ERP permiten ahorrar costos a las empresas, tanto en infraestructura física y talento humano, según el estudio realizado se manifestó la utilización de las redes sociales tales como Facebook, Instagram, Twitter, YouTube, las cuales en su conjunto son los nuevos aliados estratégicos que trabajan en coordinación con las Pymes, siendo en este sentido que el actual trabajo de investigación se desarrolló con enfoque cuantitativo, lo que permitió diagnosticar la situación actual de los emprendimientos en redes sociales y busca establecer a las tecnologías de información como una respuesta efectiva en el crecimiento de las Pymes y su transformación en empresas competitivas y emergentes.

Palabras Claves: emprendimiento; ciclo de vida; sistemas de información; competitividad; transformación.

Abstract: The present study aims to establish the characteristics of the emerging digital company, the advantages it has as an organization and the relationship of Management Information Systems with the life cycle of companies. With the new trends and advances in the use of tools such as information technologies, it is possible to predict possible scenarios that allow to establish strategies with greater degree of accuracy, which are platform of transformation of small and medium enterprises in competitive and profitable organizations, These business tools such as Business Intelligence, CRM or ERP allow companies to save costs, both in physical infrastructure and human talent, according to the study showed the use of social networks such as Facebook, Instagram, Twitter, YouTube, Which together are the new strategic allies that work in coordination with companies, by means of this research work was carried out with a quantitative approach, which allowed to diagnose the current situation of the enterprises in social networks and seeks to establish information technologies as an effective response in the growth of SMEs and their transformation into competitive and emerging companies.

Key words: entrepreneurship; life cycle; information systems; competitiveness; transformation 


\section{Introducción}

Actualmente las pequeñas y medianas empresas (PYMES) tienen un papel importante en el desarrollo productivo de América Latina, sin embargo, debido a sus dimensiones tienen una participación menor en el Producto Interno Bruto del país del cual proceden. Su producción está mayormente vinculada con el mercado interno por lo que una parte significativa de la población y de la economía de la región dependen de su actividad, en otro aspecto la participación de las pymes en "las exportaciones es bastante reducida como resultado de un escaso desempeño en términos de competitividad, que también se expresa en la marcada brecha de productividad respecto de las grandes empresas" (Ferraro Carlo, CEPAL, 2010)


Imagen 1: Pymes en Redes sociales y el uso de los sistemas de información Fuente: elaboración propia

La Imagen 1 nos permite apreciar las ventajas del gerente moderno, el cual a menudo opta por adaptarse a los cambios, siendo éste el pionero en el avance de la globalización en búsqueda de generar oportunidades y no solamente esperar a que lleguen (María Alejandra Masclef, Eduardo Juárez, 2005), la anticipación que tiene contra los cambios y la búsqueda de crear riqueza es también conocido como emprendimiento. Por ello, se vuelve importante buscar una sólida base de recursos y oportunidades que a su vez se guíen por un plan coherente (Vainrub, 2009). Las empresas pequeñas y medianas hoy en día están buscando la forma de generar utilidades y crecer de una manera rápida, pero sobre todo buscar un aliado estratégico que le ahorre costo y le permita seguir creciendo.

En nuestro país "el $68 \%$ de pymes ecuatorianas cuenta con ordenadores, el $82 \%$ tiene Internet y el 99\% accede a la Red a través de banda ancha fija" (Diario El Comercio, 2015); pero aún no se utiliza con toda profundidad el servicio, y en muy pocas ocasiones se utilizan para capacitación, contratación y videoconferencias (Diario El Comercio, 2015). En el plano estadístico es importante decir que dentro de EEUU "el 40\% de las PYMES muere dentro de los 5 años, las $2 / 3$ partes pasa a la segunda generación y tan solo el $12 \%$ sobrevive a la tercera generación" (Crespo Paulina, Universidad del Azuay, 2013). 




Imagen 2. Los Sistemas de Información y su importancia en la toma de decisiones Fuente: elaboración propia

Así como nos muestra la Imagen 2, el poder competitivo que necesita una empresa se basa en la calidad y cantidad de información, para ello existe el Business Intelligence que se conoce como un mercado que tiene como objetivo facilitar el acceso y análisis de la información corporativa y proporcionar las herramientas tecnológicas adecuadas para la toma de decisiones (Aguirre Geanina, Andrade Henry; Espol, 2006). La competencia y la oportunidad en los negocios es la mejor forma para adquirir una ventaja competitiva en el mercado. Es así que las herramientas tecnológicas permiten crear conocimiento y adquirir nuevas formas de creación de riquezas. Las Pymes entienden que:

"los productos ya sean estos bienes o servicios poseen cualidades o atributos, que amerita resaltar y dar énfasis para lograr la atención del cliente, como son las características y beneficios, esto es lo que se va a visualizar del producto y/o servicio, por esta razón la importancia al momento de difundir porque es la propagación, la acción de hacer público lo que se ofrece". (Carvajal Juliana, 2014).

Desde hace veinte años las comunidades online se han ido adaptando a los distintos servicios (y motivos) que han ido surgiendo en internet. (Carvajal Juliana, 2014). De acuerdo a la investigación, las comunidades online se basaban fundamentalmente en el uso del correo electrónico o de los grupos de noticias (Carvajal Juliana, 2014). Actualmente la evolución de la tecnología web ofrece nuevos servicios tales como las redes sociales, "que permiten crear comunidades de usuarios de una forma simple, ágil y versátil” (Carvajal Juliana, 2014)

Siguiendo la línea de investigación, "Una de cada cuatro PYMES ecuatorianas utiliza las redes sociales para su negocio, entre las principales tenemos a Facebook, Twitter, Instagram y sus aplicaciones, YouTube entre otros" (Carvajal Juliana, 2014), principalmente para promoción de sus productos, comunicación con clientes, prospectos y captura de información valiosa para el negocio.

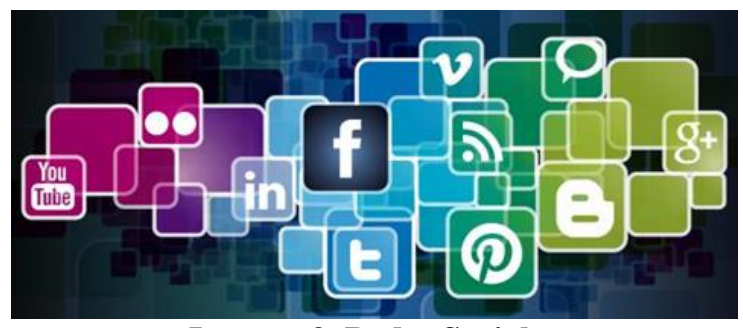

Imagen 3. Redes Sociales

Fuente: elaboración propia 
Como se observa en la imagen 3, las redes sociales crecen con rapidez, y en este sentido las Pymes que tienen sus negocios en redes sociales también se encuentran desarrollando rápidamente y permiten al emprendedor concretar negocios y expandir su marca, así nos lo indica Santiago Cordero, creador de la firma Conejo Rojo: "Las redes sociales son un gran estímulo para quienes generan sus ideas de negocios; es una forma ágil y rápida de promocionarse a nivel mundial" (ACR EL MERCURIO, 2016). En otro sentido de la investigación, el emprendedor es protagonista clave para impulsar procesos de cambio social, y destacado en los últimos años "en la comunicación de múltiples sistemas funcionales, sobre todo desde el científico, el político y el económico" (Pfeilstetter Richard, Gazeta de Antropología, 2011). Es también, según estudios realizados: "el emprendedor es el sujeto social tipo ideal que responde a todos esos aspectos centrales que definen la sociedad actual" (Pfeilstetter Richard, Gazeta de Antropología, 2011). Así también el emprendedor:

"responde mediante la construcción reflexiva, flexible y funcionalmente adecuada, de acciones racionales cuyo conjunto desemboca en una biografía única y diferenciada, capaz de enfrentar los desafíos de riesgo e incertidumbre del entorno" (Pfeilstetter Richard, Gazeta de Antropología, 2011).

Continuando con el estudio analizaremos al emprendimiento con conceptos y su relación las Pymes en la localidad.

\section{El Emprendimiento}

El emprendimiento se relaciona estrechamente con la creación de empresas, y al hablar de los modelos económicos que llevan a la creación de empresas no podemos dejar de citar el modelo emprendedor de Gartner, el vínculo entre el emprendedor y la teoría de la firma, el emprendedor y la búsqueda de oportunidades, y el modelo teórico del Programa Global Entrepreneurship Monitor (GEM). Todos estos modelos sitúan al hombre como un individuo que actúa permitiéndose y dejándose influenciar por la innovación, clave indispensable en épocas de emprendimiento. A través de la investigación encontramos estudios realizados por la Espae Graduate School of Management (2015), en donde se indica que Ecuador obtuvo un índice de Actividad Emprendedora Temprana (TEA) de 32.6\% en el 2014, lo cual indica que uno de cada tres adultos había iniciado los trámites para establecer un emprendimiento, o poseía un negocio cuya antigüedad no era mayor a los cuatro años. Dentro del estudio, también se menciona que el $66.4 \%$ de la población encuestada considera al emprendimiento como una buena opción de desarrollo profesional, el 67\% considera que el éxito en el campo de los negocios confiere estatus y respeto, y un $82.9 \%$ está de acuerdo en que el mecanismo utilizado por los medios de comunicación, al resaltar a los emprendedores, contribuye con la cultura que favorece el emprendimiento en el país.

Siguiendo este análisis, podríamos coincidir en que los individuos que tienen mayor nivel de educación se encuentran en mejores condiciones de detectar oportunidades y convertirlas en ideas de negocios, que aquellos que intenten realizar un emprendimiento por causas mayores como es la necesidad que en muchas ocasiones se ve influenciada por la falta de trabajo o un salario que no cubre lo necesario. Es importante destacar que no se evidencian resultados 
estadísticamente distintos entre las tasas de emprendimiento por necesidad relacionada al nivel de educación. (ACR EL MERCURIO, 2016)

A este análisis también se suma la proporción de emprendedores que se ubican en áreas urbanas y rurales del país, siendo así que en un estudio realizado por la ESPAE también se detecta que en año 2014 la mayor proporción de emprendedores se situó en las zonas urbanas. Para el año 2015 en el Ecuador se registra un 64\% de individuos que percibieron oportunidades, sin embargo, solo el $45 \%$ pudo establecer un negocio. Es así que existen individuos que registraron también otras percepciones, miedo al fracaso e intención de emprender (ESPAE Graduate School of Management, 2015)

Siguiendo la línea de investigación, encontramos que el Ecuador es "el segundo país que más emprende en el mundo" (Diario El Universo, Economía, 2016). En otro tema, es importante destacar que en la actualidad las PYMES, en un menor porcentaje de aprovechamiento, así también como las organizaciones globales consideran a la recolección e interpretación de la información para la toma de decisiones "como una herramienta básica de la administración en todos los niveles" (Aguirre Geanina, Andrade Henry; Espol, 2006). En la actualidad la mayoría de las organizaciones cuentan con un sistema de información que no lo utilizan en toda su capacidad, ya sea por desconocimiento o por miedo al cambio, poseen redes sociales pero su escaso intercambio de información precisa una propuesta de mejorar su utilización. Con el transcurso del tiempo estas aplicaciones llegan a tener la historia de la organización.

Utilizando la técnica del planteamiento y formulación del problema, se estableció que existe una relación entre el emprendimiento y el ciclo de vida de las pymes en redes sociales de la ciudad de Guayaquil. Entre los Síntomas que se mencionan a continuación, se estableció que:

- Las Pymes en redes sociales sobreviven un año de vida. (Diario El Comercio, 2015)

- Las redes sociales se relacionan con los índices de emprendimiento en la ciudad de Guayaquil. (Diario El Universo, Economía, 2016)

Entre las Causas que se mencionan, ratifican la relación existente entre el emprendimiento y el ciclo de vida de las Pymes en redes sociales, entre las cuales tenemos:

- Las redes sociales influyen en una empresa emergente digital, porque a través de la interacción, consumo y despacho de sus productos permite el crecimiento económico de las mismas. (Crespo Paulina, Universidad del Azuay, 2013)

- Se relacionan las redes sociales con los emprendimientos por la interacción que existe en buscar satisfacer necesidades, y la creación de nuevas oportunidades de negocios (Aguirre Geanina, Andrade Henry; Espol, 2006)

- Continuando con el análisis se describe el Pronóstico que se encontró en el presente trabajo de investigación:

- ¿Cómo debería ser el Plan de Negocios que sigan las Pymes que tienen sus operaciones en redes sociales?

- ¿Qué influencia tendrían las Pymes en redes sociales sobre el índice de emprendimiento actual?

- A través de la técnica se estableció el presente Control del Pronóstico:

- Existe en la actualidad Sistemas de Información que permiten la elaboración del plan de negocios de la empresa. 
- Las redes sociales y la globalización exigen a las empresas adoptar una postura digital que permite su crecimiento dentro del mercado.

El Pronóstico y Control del Pronóstico nos permitieron realizar la formulación del problema de investigación, que se presenta a continuación:

¿En qué medida se relaciona el ciclo de vida de las Pymes en redes sociales y el emprendimiento en la ciudad de Guayaquil?

Los objetivos de la investigación, se presentan de la siguiente manera

\section{Objetivo General}

Analizar la relación entre el ciclo de vida de las Pymes en redes sociales y el emprendimiento en la ciudad de Guayaquil.

\section{Objetivos específicos}

- Cuantificar el mercado de consumidores de redes digitales existentes en la ciudad de Guayaquil, para así establecer la relación entre el uso de redes sociales y su crecimiento en el mercado.

- Proponer a los Sistemas de Información Gerencial como una herramienta eficaz en la toma de decisiones en las Pymes.

\section{Justificación Teórica de la investigación}

El estudio surgió de la necesidad de optimizar la toma de decisiones gerenciales que existen en las Pymes, porque con el estudio se demuestra que las ausencias de buenas decisiones afectaron el ciclo de vida de las pequeñas y medianas empresas (Diario El Comercio, 2015) que existen en redes sociales. Lo cual es un indicador muy claro de la relación existente entre el emprendimiento y el ciclo de vida de las Pymes en redes sociales.

Continuando con los pasos que conlleva el análisis se presentó el esquema del método de las variables, para establecer la relación existente entre el emprendimiento y el ciclo de vida de las pymes en redes sociales, como se detalla a continuación cada uno de sus componentes:

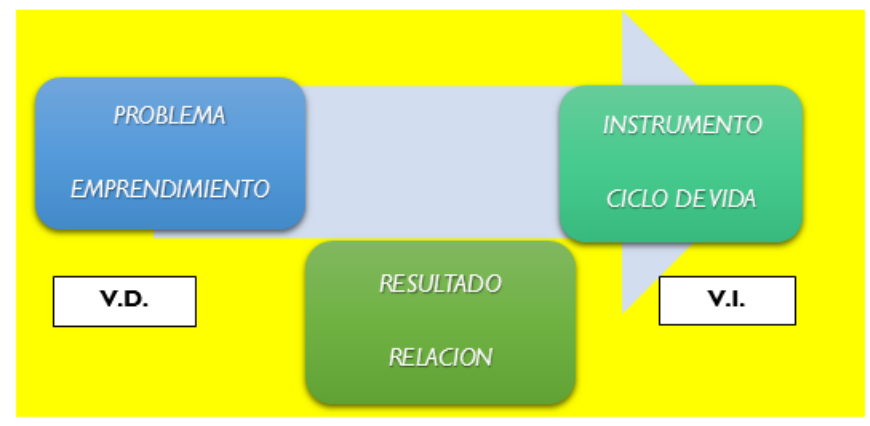

Gráfico 1. Método de las variables Fuente: elaboración propia 
En el Gráfico 1 se presentó los componentes y las variables tanto la variable independiente como la variable dependiente y el resultado que se buscó en el presente análisis.

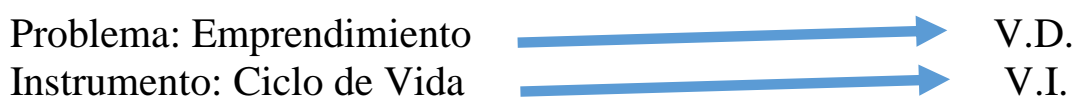

\section{Resultado: Relación}

A través de la presentación del Método de las Variables se estableció la lo siguiente:

\section{Hipótesis general}

Si las Pymes en redes sociales continúan su participación en el mercado de bienes y servicios, ¿existiría una relación entre las Pymes en redes sociales y el emprendimiento de la ciudad de Guayaquil?

Las siguientes Hipótesis Particulares colaboran con el análisis de estudio que se presentan a continuación:

- ¿Si se adopta un sistema gestión sobre la calidad de los procesos influiría sobre el ciclo de vida de las Pymes?

- ¿Si se realiza un análisis sobre los beneficios de los Sistemas de Información Gerencial en la toma de decisiones, se mejorarían los resultados de las Pymes dentro de la economía local?

A través del esquema de la formulación del problema se estableció la relación que existe entre el emprendimiento y las Pymes en redes sociales, sin embargo, es importante destacar también la participación de las herramientas tecnológicas en el crecimiento de las pequeñas y medianas empresas y que fueron descubiertas en el presente trabajo de investigación.

\section{Las Tecnologías de la Información y las Redes Sociales}

Siguiendo nuestro estudio encontramos en textos el impacto de los Sistemas de Información en el desarrollo de los negocios, es así que se rescata el pensamiento del siguiente autor:

"Un sistema de información es un organismo que recolecta, procesa, almacena y distribuye información para apoyar en la toma de decisiones y el control en una organización". (Kenneth C. Laudon, Jane P. Laudon, 2012)

Por ello, podemos destacar que una empresa emergente en el mundo contemporáneo es totalmente digital, porque los negocios se realizan las 24 horas del día y los siete días de la semana (Kenneth C. Laudon, Jane P. Laudon, 2012), entre las ventajas que se tiene se describe las siguiente:

- Las relaciones comerciales actualmente se realizan en forma digital.

- Los procesos de negocios se llevan a través de redes digitales 
- Los activos corporativos son administrados digitalmente.

\section{Los sistemas de información y su influencia en los negocios}

Basado en nuestra investigación sobre el texto del autor se determina que la implementación de Sistemas de Información permite a las empresas obtener ventajas significativas sobre su competencia (Kenneth C. Laudon, Jane P. Laudon, 2012), entre las principales tenemos:

- Tomar decisiones:

Porque se puede ofertar bienes y servicios en línea y permite realizar transacciones comerciales mediante las TIC

- Diseñar escenarios prospectivos:

Porque permite planificar sus actividades eficaces y eficientes a través del uso de métodos de escenarios que podrían pronosticar el futuro para la toma de decisiones.

Hoy existe una mayor interdependencia entre los sistemas de información de la empresa y sus herramientas de negocios (José Ramón Gallardo Hernández, 2014). Los cambios en la estrategia, las reglas y los procesos de negocios requieren cambios en el hardware, el software, las bases de datos y las telecomunicaciones.

\section{La Cadena de Valor de la Información}

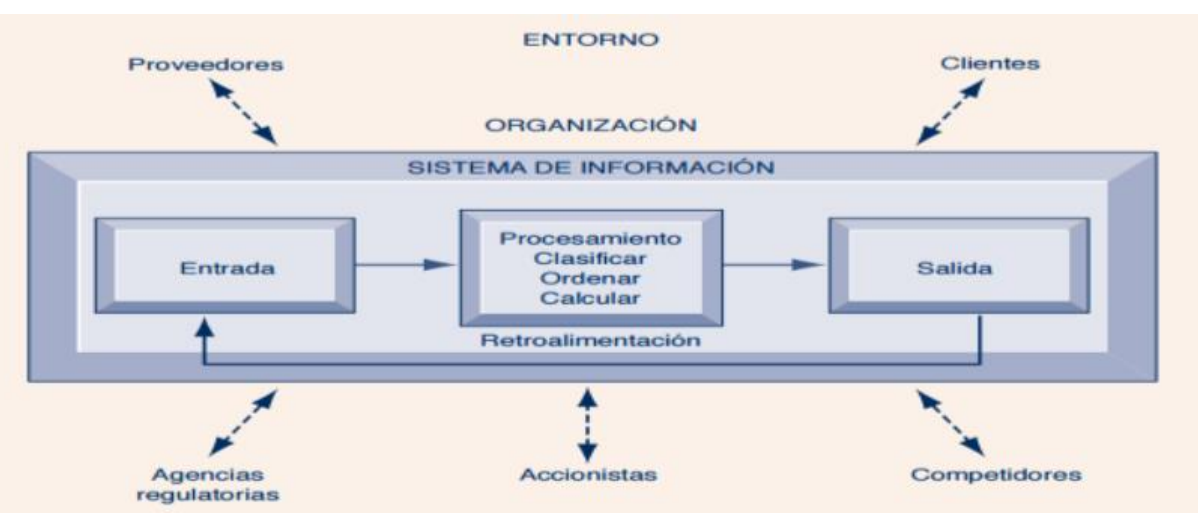

Imagen 4. La Cadena de Valor de la Información

Fuente: Laudon, Kenneth; Laudon, Jane. "Sistemas de Información Gerencial (Décimo segunda Edición)”, Pearson, 2012, México.

De acuerdo a lo que se indica en la imagen 4, "un sistema de información contiene datos sobre una organización y el entorno que la rodea" (Kenneth C. Laudon, Jane P. Laudon, 2012). Este proceso realiza tres actividades básicas (entrada, procesamiento y salida) y producen la información que necesitan las empresas. (Kenneth C. Laudon, Jane P. Laudon, 2012). La retroalimentación es la salida que se devuelve a las personas o actividades apropiadas en la organización para evaluar y refinar la entrada. (Kenneth C. Laudon, Jane P. Laudon, 2012)

Una vivienda es una analogía muy parecida, las casas de las personas se construyen con martillos, clavos y madera, pero no solo esto las constituye (Idalberto Chianvenato, 2009), es 
necesario la arquitectura, el diseño, el entorno, el paisaje y todas las decisiones que conducen a la creación de estas características son las que "en su conjunto forman parte de la casa y cruciales para resolver el problema de poner un techo sobre la cabeza de nosotros" (Kenneth C. Laudon, Jane P. Laudon, 2012)

Siguiendo la línea de investigación se encontró la existencia de un porcentaje muy grande de la población que utiliza redes sociales en la ciudad de Guayaquil (Ver Tabla 1), lo cual permitirá establecer una propuesta de implementación de un plan de gestión que busque consolidar el crecimiento y participación de las Pymes en redes sociales.

\section{Población con celular y redes sociales}

En la tabla 1 se muestra el portal del INEC, en el cual se detalla el crecimiento de la población que gusta utilizar medios tecnológicos y redes sociales.

Tabla 1. Población con celular y Redes Sociales

\begin{tabular}{|l|r|r|r|r|r|r|}
\hline Desagregación & \multicolumn{1}{c|}{2011} & \multicolumn{1}{c|}{2012} & \multicolumn{1}{c|}{2013} & \multicolumn{1}{c|}{2014} & \multicolumn{1}{c|}{2015} & \multicolumn{1}{c|}{2016} \\
\hline Porblación Total & \multicolumn{1}{c|}{14.478 .129} & 14.682 .556 & 15.872 .755 & 16.148 .648 & 16.404 .531 & 16.714 .929 \\
\hline Población de 5 años y más & 10.533 .003 & 10.864 .147 & 11.200 .371 & 11.159 .255 & 14.746 .884 & 15.114 .789 \\
\hline Tiene teléfono celular activado & 6.209 .858 & 6.859 .938 & 7.453 .781 & 7.820 .597 & 8.174 .520 & 8.482 .236 \\
\hline El teléfono es SMARTPHONE & 522.640 & 839.705 & 1.261 .944 & 1.928 .108 & 3.084 .886 & 4.484 .087 \\
\hline En su teléfono utiliza redes sociales & 365.427 & 641.914 & 1.081 .620 & 1.722 .159 & 2.807 .282 & 4.224 .984 \\
\hline
\end{tabular}

Fuente: http://www.ecuadorencifras.gob.ec//tecnologias-de-la-informacion-y-comunicacion-tic/

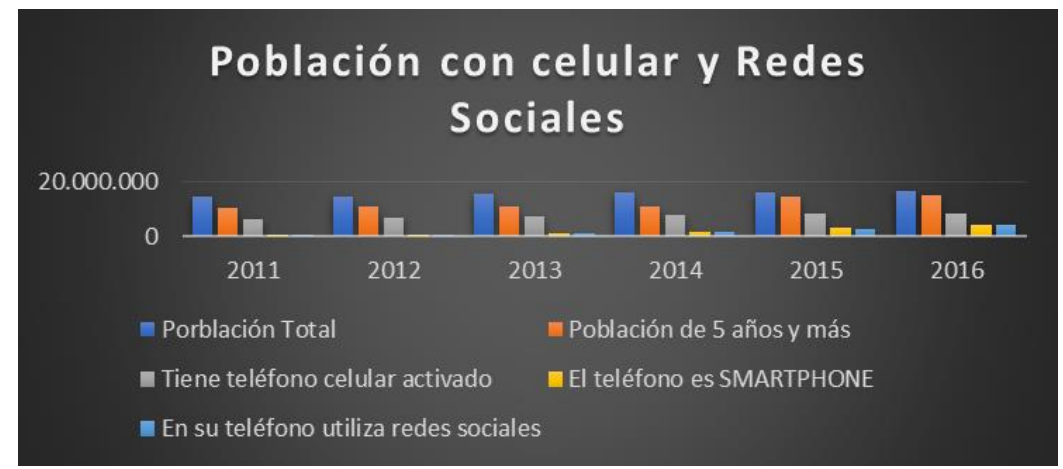

Gráfico 2. Población con celular y Redes Sociales

Fuente: http://www.ecuadorencifras.gob.ec//tecnologias-de-la-informacion-y-comunicacion-tic/

En el Gráfico 2 se presenta la existencia de la población que utiliza herramientas tecnológicas y redes sociales, los cuales son para el presente estudio los posibles consumidores de bienes y servicios producidos por las pequeñas y medianas empresas.

\section{Pymes en Redes Sociales y las tecnologías de la Información}

Las herramientas tales como los CRM, los ERP, el uso adecuado de Business Intelligence han ayudado a las empresas a mejorar su rentabilidad, a través de mejorar en las estrategias a implementar, el apoyo en la toma de decisiones, (Diario El Comercio, 2015) 


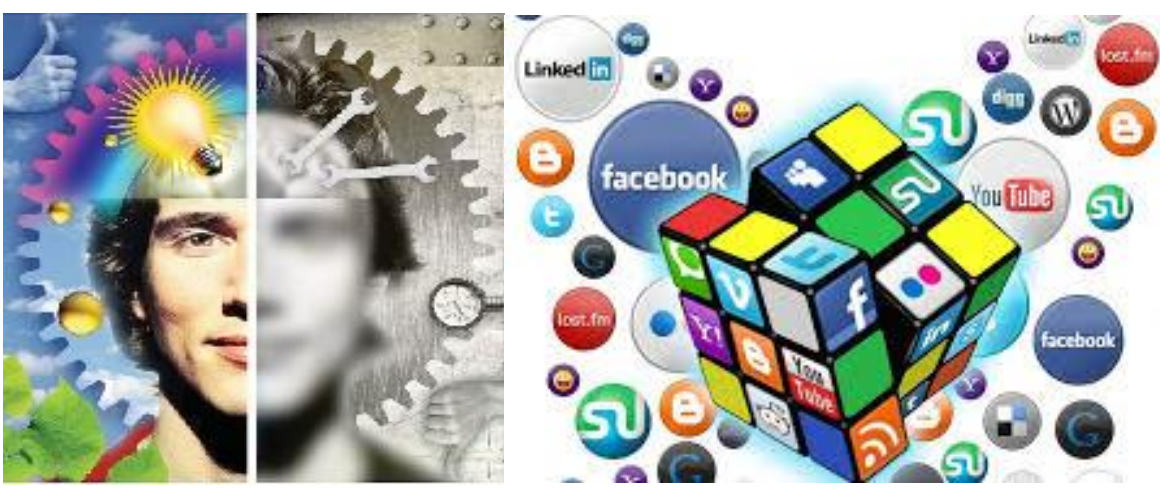

Imagen 5. Las Pymes en redes sociales Fuente: elaboración propia

Tal como nos muestra la Imagen 5, existen redes sociales preferidas por las empresas, que entre ellas tenemos: Facebook, Twitter, YouTube, Google+ y LinkedIn. "La popularidad de las redes sociales ha abierto para las empresas un nuevo canal de comunicación, promoción y venta; la pregunta es: ¿cómo utilizarlo de manera efectiva?” (Ekos, Investigin.com, 2015)

Los negocios pueden abrir una cuenta, pero no todas obtienen beneficios reales, para lograr los objetivos es necesario entender el alcance y lo que se puede hacer con las redes sociales, de acuerdo con la naturaleza de la empresa (Ekos, Investigin.com, 2015).

\section{Conclusiones y Recomendaciones}

El presente estudio permitió establecer que existe una relación entre el emprendimiento y el ciclo de vida de las Pymes en redes sociales, de los cuales indica que existe un $28 \%$ de pequeñas y medianas empresas que utilizan redes sociales, para realizar operaciones comerciales (ACR EL MERCURIO, 2016) y, “existe también un 37\% de Pymes que lo utilizan como medio de información" (Diario El Comercio, 2015). Esto se sintetiza en las siguientes ideas principales que concluyen el presente estudio:

- En un medio altamente competitivo y globalizado es una necesidad de primera mano optar por un sistema de gestión que puede ser representado por un CRM, ERP y utilización de Business Intelligence, como herramientas de negocios que busquen mejorar la participación en el mercado de las Pymes y la consolidación de las relaciones con sus clientes.

- Existe una relación entre el emprendimiento y el ciclo de vida de las Pymes, la cual representa dentro de la economía un 37\% de participación en el mercado, creando riqueza, nuevas fuentes de empleo seguro, la búsqueda de nuevas tecnologías también optimiza el crecimiento de las Pymes en el mercado.

Como recomendaciones podemos referir las siguientes:

\section{Elegir el mejor canal}

- Cada red social tiene su propia personalidad. Facebook puede ser útil para mantener la comunicación más cercana con los clientes y mostrar los productos y servicios de la 
empresa. Twitter en cambio es ideal para servicios que requieren rápida atención o noticias coyunturales. Adicional YouTube es excelente para mostrar un tutorial para usar un producto o revelar una campaña. (Ekos, Investigin.com, 2015)

\section{Hablar al público objetivo}

- Las redes sociales crean la oportunidad de hablar con personas que se interesan en los productos, que desean consumirlos y en ocasiones sugieren mejoras. Existen empresas que utilizan un nicho específico. Conocer bien el público que quiere atrapar le permitirá enviar mensajes más efectivos (Ekos, Investigin.com, 2015).

\section{Brindar atención a sus clientes}

- Los clientes perciben una cercanía entre empresa y ellos a través de las redes sociales, un cliente que recibe una rápida respuesta, se podría convertir en un cliente frecuente.

\section{Vender la marca, y los productos solo de manera indirecta}

- Le conviene al productor y dueño de la mercadería darle una personalidad a su marca (Ekos, Investigin.com, 2015). Hablar sobre los valores de la empresa, la visión y misión sirve mucho.

\section{Contratar un experto}

- Los negocios las redes sociales son el principal canal de venta, promoción y atención a clientes. Por ello es tan importante que un especialista en social media, desarrollo de contenidos o marketing colabore con usted para crear una estrategia efectiva (Ekos, Investigin.com, 2015)

\section{Bibliografía}

Acr El Mercurio. (8 de 11 de 2016). Emprendimiento que se mueve vía redes sociales, pág. 1.

Aguirre Geanina, Andrade Henry; Espol. (2006). BI Business Intelligence. Guayaquil: Espol.

Carvajal Juliana, P. A. (2014). Análisis De Las Redes Sociales Y Su Incidencia En Las Ventas De Las Pymes. Milagro: Repositorio, Universidad Estatal De Milagro.

Crespo Paulina, Universidad del Azuay. (2013). El uso de las TICS como herramienta para la internacionalización de las PYMES en el Ecuador. Cuenca: Repositorio Universidad del Azuay.

Diario El Comercio. (19 de Julio de 2015). El 82\% de pymes de Ecuador accede a Internet, pero su uso se limita a enviar correos y tareas administrativas, pág. 1.

Diario El Universo, Economía. (1 de Junio de 2016). Ecuador es el segundo país que más emprende en el mundo, pero los negocios no se consolidan, pág. 1.

Ekos, Investigin.com. (2015). Pymes: 5 claves para utilizar las redes sociales. EKOS, 2. 
ESPAE Graduate School of Management. (2015). Gem Ecuador 2015 Report. Guayaquil: Gem Ecuador.

Ferraro Carlo, CEPAL. (2010). Políticas de apoyo a las pymes en América Latina. Santiago de Chile: CEPAL.

Idalberto Chianvenato. (2009). Comportamiento Organizacional, la dinámica del éxito en las organizaciones. México, DF: Mc Graw Hill.

José Ramón Gallardo Hernández. (2014). Administración Estratégica, de la visión a la ejecución. México: Alfaomega.

Kenneth C. Laudon, Jane P. Laudon. (2012). Sistemas de Información Gerencial. México: PEARSON.

María Alejandra Masclef, Eduardo Juárez. (2005). Tecnología para Ejecutivos. Buenos Aires: Recoletos Argentina S.A.

Miguel Angel Bazzano. (2007). Experiencia de Empresas Argentinas. Revista de ciencias de la Administración en implementación de Sistemas de Información, 18.

Pfeilstetter Richard, Gazeta de Antropología. (2011). El emprendedor. Una reflexión crítica sobre usos y significados actuales de un concepto. Gazeta de Antropología, 15.

Vainrub, R. (2009). Una guía para emprendedores. Ciudad de México: Pearson.

Bibliografía de imágenes y gráficos http://www.ecuadorencifras.gob.ec//tecnologias-de-lainformacion-y-comunicacion-tic/ 